\title{
RELIABILITY ASSESSMENT FOR A FOUR CYLINDER DIESEL ENGINE BY EMPLOYING ALGEBRA OF LOGICS
}

\author{
Reena Garg \\ Assistant Professor, YMCA University, Faridabad, India
}

\begin{abstract}
In this paper, the authors have evaluated reliability of four cylinder diesel engine by employing the Algebra of logics which is easier in comparison of old techniques. Here, a multi-component fuel system in diesel engine, comprised of four subsystems in series, has considered. The authors, in this model, have considered a parallel redundant fuel injective device to improve the system's performance. The whole system can fail due to failure of atleast one component of all the routes of flow.
\end{abstract}

Boolean function technique has used to formulate and solve the mathematical model. Reliability and M.T.T.F. of the considered diesel engine have been obtained to connect the model with physical situations. A numerical example and its graphical representation have been appended at last to highlight important results.

Keywords: Boolean function, Algebra of logics, Parallel redundancy, Four cylinder diesel engine, Weibull time distribution, Exponential time distribution, Reliability, MTTF etc.

\section{INTRODUCTION:}

An unreliable system has higher probability of failure [1] as and when any item of the associated equipment is damaged or destroyed due to some causes. Consequently, the unreliability of the system [2] may result into wastage of cost, time of personal as well as national security. Thus, in conclusion, a high degree of reliability is prime requirement in various practical fields e.g., nuclear power plant, space systems and defense systems etc. So, it is necessary to evaluate reliability and other performance measures in advance to avoid above mentioned losses. The previous methods [3-6] of obtaining reliability have tedious calculations and are so typical. So, in this paper, the authors have evaluated reliability of four cylinder diesel engine by employing the Boolean function technique [8] which is easier in comparison of old techniques. Fuel system, discussed here helps to transfer the fuel from the fuel tank to the engine and regulate its flow into the cylinders depending on speed and local requirements. The system configuration has shown in fig-1. In this system the fuel is injected into the cylinders using an injection pump and injectors. The diesel fuel from the tank is pump to the fuel injection device. This device consists of small plunger pump operating to push the fuel through the injectors (nozzles) into the cylinders and one can operates one pump from the fuel injection pumps at the appropriate time i.e., towards the end of the compression stroke the fuel is pushed through the small hole in the injector and is atomized as it enters into the cylinder.

\section{ASSUMPTIONS:}

Following assumptions have been taken care this model:

(i) Initially, all components of the system are good.

(ii) Each component of the system remains either in good or in bad state.

(iii) There is no repair facility available.

(iv) Failure times of all the components of the system are arbitrary.

(v) All the states of different components are statistically independent.

(vi) Reliability of each component of the system is known in advance.

(vii) The diesel engine as a whole can fail, if atleast one component in all the routes of flow fails.

(viii) Transition from one component to other is hundred percent reliable.

\section{NOMANCLATURE:}

Used nomenclature in this study is as follows:

$\begin{array}{lc}x_{1} & : \text { State of diesel tank. } \\ x_{2} & : \text { State of transfer pump. } \\ x_{3}, x_{4} & : \text { States of fuel injective devices. } \\ x_{5}, x_{6}, x_{7}, x_{8} & : \text { States of cylinders. } \\ x_{i}^{\prime}(i=1,2--8) & : \text { Negation of } x_{i} . \\ x_{i}(i=1,2--8) & : 1 \text { in good state; } 0 \text { in bad state. }\end{array}$




\section{$\wedge /$ : Conjunction /disjunction.}

$R_{i}(i=1,2--8): \quad$ Reliability of the component corresponding to state $x_{i}$

$Q_{i} \quad:$ is $\left(1-R_{i}\right) ; \forall i=1,2--8$

$R_{S} \quad$ : Reliability of the system as a whole.

$\mid$ |: This sign has used to represent logical matrix.

\section{FORMULATION OF MATHEMATICAL PROBLEM:}

Using Boolean function technique, the conditions of capability of the successful operation of the diesel engine in terms of logical matrix are expressed as:

$$
D\left(x_{1}, x_{2}--, x_{8}\right)=\left|\begin{array}{llll}
x_{1} & x_{2} & x_{3} & x_{5} \\
x_{1} & x_{2} & x_{3} & x_{6} \\
x_{1} & x_{2} & x_{3} & x_{7} \\
x_{1} & x_{2} & x_{3} & x_{8} \\
x_{1} & x_{2} & x_{4} & x_{5} \\
x_{1} & x_{2} & x_{4} & x_{6} \\
x_{1} & x_{2} & x_{4} & x_{7} \\
x_{1} & x_{2} & x_{4} & x_{8}
\end{array}\right|
$$

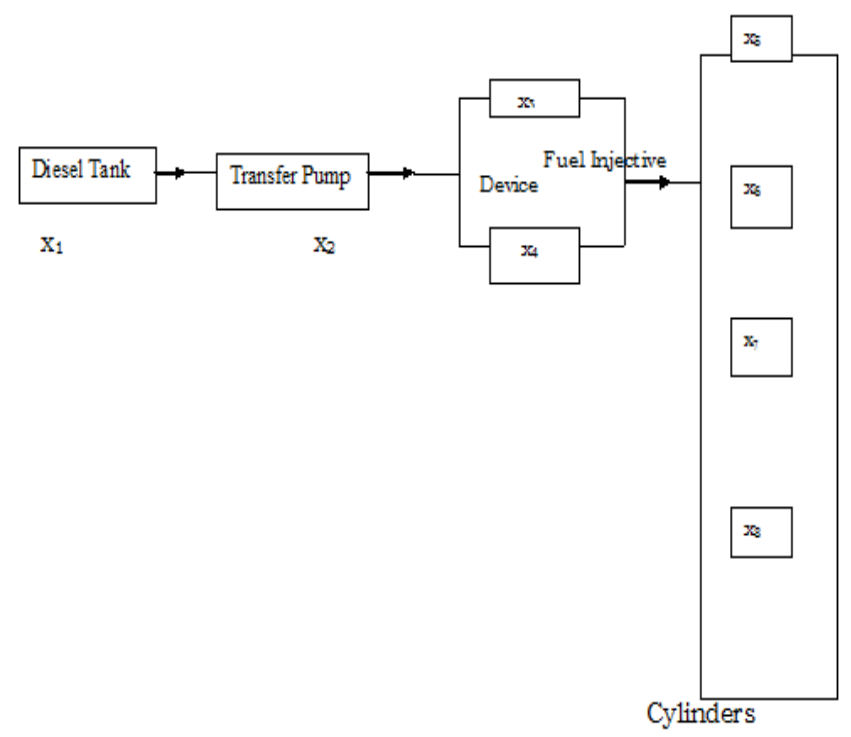

\section{SOLUTION OF THE PROBLEM:}

By making use of algebra of logic, we may rewrite equation (1) as:

$$
D\left(x_{1}, x_{2}--, x_{8}\right)=\left|\begin{array}{ll}
x_{1} & x_{2} \wedge d\left(x_{1}, x_{2}----x_{8}\right)
\end{array}\right|
$$

Where,

$$
d\left(x_{1}, x_{2}---x_{8}\right)=\left|\begin{array}{cc}
x_{3} & x_{5} \\
x_{3} & x_{6} \\
x_{3} & x_{7} \\
x_{3} & x_{8} \\
x_{4} & x_{5} \\
x_{4} & x_{6} \\
x_{4} & x_{7} \\
x_{4} & x_{8}
\end{array}\right|
$$

Substituting

$$
\begin{aligned}
& A_{1}=\left|\begin{array}{ll}
x_{3} & x_{5}
\end{array}\right| \\
& A_{2}=\left|\begin{array}{ll}
x_{3} & x_{6}
\end{array}\right| \\
& A_{3}=\left|\begin{array}{ll}
x_{3} & x_{7}
\end{array}\right| \\
& A_{4}=\left|\begin{array}{ll}
x_{3} & x_{8}
\end{array}\right| \\
& A_{5}=\left|\begin{array}{ll}
x_{4} & x_{5}
\end{array}\right| \\
& A_{6}=\left|\begin{array}{ll}
x_{4} & x_{6}
\end{array}\right| \\
& A_{7}=\left|\begin{array}{ll}
x_{4} & x_{7}
\end{array}\right|
\end{aligned}
$$

And

$$
A_{8}=\left|\begin{array}{ll}
x_{4} & x_{8}
\end{array}\right|
$$

These values in equation (3), one obtains:

Fig-1 (System Configuration) 


$$
d\left(x_{1}, x_{2},----x_{8}\right)=\left|\begin{array}{c}
A_{1} \\
A_{2} \\
A_{3} \\
A_{4} \\
A_{5} \\
A_{6} \\
A_{7} \\
A_{8}
\end{array}\right|
$$

Using orthogonalisation algorithm [9], equation (12) may be written as:

$$
d\left(x_{1}, x_{2},------x_{8}\right)=
$$

$$
\left|\begin{array}{lllllllll}
A_{1} & & & & & & & \\
A_{1}^{\prime} & A_{2} & & & & & & \\
A_{1}^{\prime} & A_{2}^{\prime} & A_{3} & & & & & \\
A_{1}^{\prime} & A_{2}^{\prime} & A_{3}^{\prime} & A_{4} & & & & \\
A_{1}^{\prime} & A_{2}^{\prime} & A_{3}^{\prime} & A_{4}^{\prime} & A_{5} & & & \\
A_{1}^{\prime} & A_{2}^{\prime} & A_{3}^{\prime} & A_{4}^{\prime} & A_{5}^{\prime} & A_{6} & & \\
A_{1}^{\prime} & A_{2}^{\prime} & A_{3}^{\prime} & A_{4}^{\prime} & A_{5}^{\prime} & A_{6}^{\prime} & A_{7} & \\
A_{1}^{\prime} & A_{2}^{\prime} & A_{3}^{\prime} & A_{4}^{\prime} & A_{5}^{\prime} & A_{6}^{\prime} & A_{7}^{\prime} & A_{8} \\
& & & & & & &
\end{array}\right|
$$

Now, we have

$$
\begin{array}{ll}
A_{i}^{\prime}=\left|\begin{array}{cc}
x_{3}^{\prime} & \\
x_{3} & x_{\mathrm{i}+4}^{\prime}
\end{array}\right| & ; i=1,2,3 \text { and } 4 \\
A_{j}^{\prime}=\left|\begin{array}{ll}
x_{4}^{\prime} & \\
x_{4} & x_{\mathrm{j}}^{\prime}
\end{array}\right| & ; j=5,6,7 \text { and } 8
\end{array}
$$

Using algebra of logics [7], one can obtain the following results:

$$
A_{1}^{\prime} \quad A_{2}=\left|\begin{array}{ll}
x_{3}^{\prime} & \\
x_{3} & x_{5}
\end{array}\right| \wedge\left|\begin{array}{ll}
x_{3} & x_{6}
\end{array}\right|=\left|\begin{array}{lll}
x_{3} & x_{5}^{\prime} & x_{6}
\end{array}\right|
$$

Similarly, we get

$$
A_{1}^{\prime} A_{2}^{\prime} A_{3}=\left|\begin{array}{llll}
x_{3} & x_{5}^{\prime} & x_{6}^{\prime} & x_{7}
\end{array}\right|
$$

$$
\begin{aligned}
& A_{1}^{\prime} A_{2}^{\prime} A_{3}^{\prime} A_{4}=\left|\begin{array}{lllll}
x_{3} & x_{5}^{\prime} & x_{6}^{\prime} & x_{7}^{\prime} & x_{8}
\end{array}\right| \\
& A_{1}^{\prime} A_{2}^{\prime} A_{3}^{\prime} A_{4}^{\prime} A_{5}=\left|\begin{array}{llll}
x_{3}^{\prime} & x_{4} & x_{5}
\end{array}\right| \\
& A_{1}^{\prime} A_{2}^{\prime} A_{3}^{\prime} A_{4}^{\prime} A_{5}^{\prime} A_{6}=\left|\begin{array}{llll}
x_{3}^{\prime} & x_{4} & x_{5}^{\prime} & x_{6}
\end{array}\right| \\
& A_{1}^{\prime} A_{2}^{\prime} A_{3}^{\prime} A_{4}^{\prime} A_{5}^{\prime} A_{6}^{\prime} A_{7}=\left|\begin{array}{lllll}
x_{3}^{\prime} & x_{4} & x_{5}^{\prime} & x_{6}^{\prime} & x_{7}
\end{array}\right|
\end{aligned}
$$

$A_{1}^{\prime} A_{2}^{\prime} A_{3}^{\prime} A_{4}^{\prime} A_{5}^{\prime} A_{6}^{\prime} A_{7}^{\prime} A_{8}=\left|\begin{array}{llllll}x_{3}^{\prime} & x_{4} & x_{5}^{\prime} & x_{6}^{\prime} & x_{7}^{\prime} & x_{8}\end{array}\right|$

Using the equations (16) through (22), equation (13) becomes

$$
d\left(x_{1}, x_{2},---, x_{8}\right)=\left|\begin{array}{cccccc}
x_{3} & x_{5} & & & & \\
x_{3} & x_{5}^{\prime} & x_{6} & & \\
x_{3} & x_{5}^{\prime} & x_{6}^{\prime} & x_{7} & \\
x_{3} & x_{5}^{\prime} & x_{6}^{\prime} & x_{7}^{\prime} & x_{8} \\
x_{3}^{\prime} & x_{4} & x_{5} & & & \\
x_{3}^{\prime} & x_{4} & x_{5}^{\prime} & x_{6} & & \\
x_{3}^{\prime} & x_{4} & x_{5}^{\prime} & x_{6}^{\prime} & x_{7} & \\
x_{3}^{\prime} & x_{4} & x_{5}^{\prime} & x_{6}^{\prime} & x_{7}^{\prime} & x_{8}
\end{array}\right|
$$

By making use of equation (23) equations (2) gives:

$$
D\left(x_{1}, x_{2},---, x_{8}\right)=\left|\begin{array}{llllllll}
x_{1} & x_{2} & x_{3} & x_{5} & & & \\
x_{1} & x_{2} & x_{3} & x_{5}^{\prime} & x_{6} & & & \\
x_{1} & x_{2} & x_{3} & x_{5}^{\prime} & x_{6}^{\prime} & x_{7} & & \\
x_{1} & x_{2} & x_{3} & x_{5}^{\prime} & x_{6}^{\prime} & x_{7}^{\prime} & x_{8} & \\
x_{1} & x_{2} & x_{3}^{\prime} & x_{4} & x_{5} & & & \\
x_{1} & x_{2} & x_{3}^{\prime} & x_{4} & x_{5}^{\prime} & x_{6} & & \\
x_{1} & x_{2} & x_{3}^{\prime} & x_{4} & x_{5}^{\prime} & x_{6}^{\prime} & x_{7} & \\
x_{1} & x_{2} & x_{3}^{\prime} & x_{4} & x_{5}^{\prime} & x_{6}^{\prime} & x_{7}^{\prime} & x_{8}
\end{array}\right|
$$

Since, R.H.S. of equation (24) is disjunction of pair-wise disjoint conjunctions, therefore, reliability of considered diesel engine will be:

$$
R_{s}=P_{r}\left\{D\left(x_{1}, x_{2},---x_{8}\right)=1\right\}
$$




$$
\begin{aligned}
& =\mathrm{R}_{1} R_{2}\left[R_{3} R_{5}+Q_{5} R_{3} R_{6}+Q_{5} Q_{6} R_{3} R_{7}+Q_{5} Q_{6} Q_{7} R_{3} R_{8}+Q_{3} R_{4} R_{5}+Q_{3} Q_{5} R_{4} R_{6}+Q_{3} Q_{5} Q_{6} R_{4} R_{7}+Q_{3} Q_{5} Q_{6} Q_{7} R_{4} R_{8}\right. \\
& =\mathrm{R}_{1} R_{2}\left[R_{3} R_{5}+R_{3} R_{6}+R_{3} R_{7}+R_{3} R_{5} R_{6} R_{7}+R_{3} R_{8}+R_{3} R_{5} R_{6} R_{8}+R_{3} R_{6} R_{7} R_{8}\right. \\
& +R_{3} R_{5} R_{7} R_{8}+R_{4} R_{5}+R_{4} R_{6}+R_{3} R_{4} R_{5} R_{6}+R_{4} R_{7}+R_{3} R_{4} R_{5} R_{7}+R_{4} R_{5} R_{6} R_{7} \\
& +R_{3} R_{4} R_{6} R_{7}+R_{4} R_{8}+R_{3} R_{4} R_{5} R_{6} R_{7} R_{8}+R_{3} R_{4} R_{5} R_{8}+R_{3} R_{4} R_{6} R_{8}+R_{3} R_{4} R_{7} R_{8}+R_{4} R_{5} R_{6} R_{8} \\
& +R_{4} R_{5} R_{7} R_{8}+R_{4} R_{6} R_{7} R_{8}-R_{3} R_{5} R_{6}-R_{3} R_{5} R_{7}-R_{3} R_{6} R_{7}-R_{3} R_{5} R_{8}-R_{3} R_{6} R_{8}-R_{3} R_{7} R_{8} \\
& -R_{3} R_{5} R_{6} R_{7} R_{8}-R_{3} R_{4} R_{5}-R_{3} R_{4} R_{6}-R_{3} R_{5} R_{6}-R_{3} R_{4} R_{7}-R_{4} R_{5} R_{7}-R_{4} R_{6} R_{7}-R_{3} R_{4} R_{5} R_{6} R_{7} \\
& -R_{3} R_{4} R_{8}-R_{4} R_{5} R_{8}-R_{4} R_{6} R_{8}-R_{4} R_{7} R_{8}-R_{3} R_{4} R_{5} R_{6} R_{8}-R_{3} R_{4} R_{6} R_{7} R_{8} \\
& \left.-R_{3} R_{4} R_{5} R_{7} R_{8}-R_{4} R_{5} R_{6} R_{7} R_{8}\right]
\end{aligned}
$$

Where, $\quad R_{i}(i=1,2--8)$ and $\quad Q_{i}(i=1,2--8)$ are reliability and unreliability of the components of diesel engine corresponding to the states $x_{i}(i=1,2--8)$, respectively.

\section{SOME PARTICULAR CASES:}

Case I: If the Reliability of Every Component of Diesel Engine is R:

In this case, equation (25) yields:

$$
R_{S}=8 R^{4}-16 R^{5}+14 R^{6}-6 R^{7}+R^{8}
$$

\section{Case II: When Failure Rates Follow Weibull \\ Distribution}

Let $\mathrm{a}_{i}$ be the failure rate corresponding to component state $x_{i}, \forall i=1,2--8$, then reliability of diesel engine at instant ' $\mathrm{t}$ ', is given by:

$$
R_{s w}(t)=\sum_{i=1}^{23} \exp \cdot\left\{-\alpha_{i} t^{b}\right\}-\sum_{j=1}^{22} \exp \cdot\left\{-\beta_{j} t^{b}\right\}
$$

Where $b$ is a positive parameter; and :

$$
\begin{gathered}
\alpha_{1}=\mathrm{c}+a_{3}+a_{5} \\
\alpha_{2}=\mathrm{c}+a_{3}+a_{6} \\
\alpha_{3}=\mathrm{c}+a_{3}+a_{7} \\
\alpha_{4}=\mathrm{c}+a_{3}+a_{5}+a_{6}+a_{7} \\
\alpha_{5}=\mathrm{c}+a_{3}+a_{8}
\end{gathered}
$$

$$
\begin{gathered}
\alpha_{6}=\mathrm{c}+a_{3}+a_{5}+a_{6}+a_{8} \\
\alpha_{7}=\mathrm{c}+a_{3}+a_{6}+a_{7}+a_{8} \\
\alpha_{8}=\mathrm{c}+a_{3}+a_{5}+a_{7}+a_{8} \\
\alpha_{9}=\mathrm{c}+a_{4}+a_{5} \\
\alpha_{10}=\mathrm{c}+a_{4}+a_{6} \\
\alpha_{11}=\mathrm{c}+a_{3}+a_{4}+a_{5}+a_{6} \\
\alpha_{12}=\mathrm{c}+a_{4}+a_{7} \\
\alpha_{13}=\mathrm{c}+a_{3}+a_{4}+a_{5}+a_{7} \\
\alpha_{14}=\mathrm{c}+a_{4}+a_{5}+a_{6}+a_{7} \\
\alpha_{15}=\mathrm{c}+a_{3}+a_{4}+a_{6}+a_{7} \\
\alpha_{16}=\mathrm{c}+a_{4}+a_{8}
\end{gathered}
$$

$$
\begin{gathered}
\alpha_{17}=\mathrm{c}+a_{3}+a_{4}+a_{5}+a_{6}+a_{7}+a_{8} \\
\alpha_{18}=\mathrm{c}+a_{3}+a_{4}+a_{5}+a_{8} \\
\alpha_{19}=\mathrm{c}+a_{3}+a_{4}+a_{6}+a_{8} \\
\alpha_{20}=\mathrm{c}+a_{3}+a_{4}+a_{7}+a_{8} \\
\alpha_{21}=\mathrm{c}+a_{4}+a_{5}+a_{6}+a_{8}
\end{gathered}
$$




$$
\begin{aligned}
& \alpha_{22}=\mathrm{c}+a_{4}+a_{5}+a_{7}+a_{8} \\
& \alpha_{23}=\mathrm{c}+a_{4}+a_{6}+a_{7}+a_{8}
\end{aligned}
$$

Also,

$$
\begin{gathered}
\beta_{1}=\mathrm{c}+a_{3}+a_{5}+a_{6} \\
\beta_{2}=\mathrm{c}+a_{3}+a_{5}+a_{7} \\
\beta_{3}=\mathrm{c}+a_{3}+a_{6}+a_{7} \\
\beta_{4}=\mathrm{c}+a_{3}+a_{5}+a_{8} \\
\beta_{5}=\mathrm{c}+a_{3}+a_{6}+a_{8} \\
\beta_{6}=\mathrm{c}+a_{3}+a_{7}+a_{8} \\
\beta_{7}=\mathrm{c}+a_{3}+a_{5}+a_{6}+a_{7}+a_{8} \\
\beta_{8}=\mathrm{c}+a_{3}+a_{4}+a_{5} \\
\beta_{9}=\mathrm{c}+a_{3}+a_{4}+a_{6} \\
\beta_{10}=\mathrm{c}+a_{3}+a_{5}+a_{6} \\
\beta_{19}=\mathrm{c}+a_{3}+a_{4}+a_{5}+a_{6}+a_{8}+a_{4}+a_{6}+a_{7}+a_{8} \\
\beta_{11}=\mathrm{c}+a_{3}+a_{4}+a_{7} \\
\beta_{12}=\mathrm{c}+a_{4}+a_{5}+a_{7} \\
\beta_{13}=\mathrm{c}+a_{4}+a_{6}+a_{7} \\
\beta_{15}=\mathrm{c}+a_{3}+a_{4}+a_{5}+a_{6}+a_{7} \\
\beta_{16}=\mathrm{c}+a_{3}+a_{4}+a_{8}+a_{5}+a_{8} \\
\beta_{14}+a_{4}+a_{6}+a_{8} \\
\beta_{13}+a_{7}+a_{8} \\
\beta_{15}
\end{gathered}
$$

$$
\begin{gathered}
\beta_{21}=\mathrm{c}+a_{3}+a_{4}+a_{5}+a_{7}+a_{8} \\
\beta_{22}=\mathrm{c}+a_{4}+a_{5}+a_{6}+a_{7}+a_{8} \\
\text { and } c=a_{1}+a_{2}
\end{gathered}
$$

\section{Case III: When Failures Follow Exponential Time}

\section{Distribution:}

Exponential distribution is nothing but a particular case of Weibull distribution for $b=1$ and is very useful for the practical problem purpose. Therefore, the reliability of considered diesel engine as a whole at an instant ' $t$ ', is expressed as:

$$
R_{S E}(t)=\sum_{i=1}^{23} \exp \cdot\left\{-\alpha_{i} t\right\}-\sum_{j=1}^{22} \exp \cdot\left\{-\beta_{j} t\right\}
$$

The expression for M.T.T.F., in this case, is given by

$$
\begin{aligned}
\text { M.T.T.F. } & =\int_{0}^{\infty} R_{S E}(t) \mathrm{dt} \\
& =\sum_{i=1}^{23}\left\{\frac{1}{\alpha_{i}}\right\}-\sum_{j=1}^{22}\left\{\frac{1}{\beta_{j}}\right\}
\end{aligned}
$$

\section{NUMERICAL COMPUTATION}

For a numerical computation, let us set the values:

(a) $a_{i}(i=1,2,-, 8)=a=0.04, b=2$ and $\mathrm{t}=0,1,2$ - in equation (27);

(b) $a_{i}(i=1,2,-, 8)=a=0.04$ and $\mathrm{t}=0,1,2---$ in equation (28);

(c) $a_{i}(i=1,2,-, 8)=a=0,0.1,0.2 \ldots \ldots-\ldots$ in equation (29).

By using (a) and (b), one may compute the table- 1 and by using (c) the table 2. Corresponding graphs have shown through fig. 2 and 3 , respectively.

\section{CONCLUSIONS}

In this paper, the authors have considered a four cylinder diesel engine for its reliability and M.T.T.F. evaluation by using Boolean function technique. This technique is easier than the other known techniques for reliability evaluation and used only for non-repairable systems. The author's idea is to taking one parallel redundant fuel injective device to improve system's performance. Reliability of the whole system has computed in three different cases. M.T.T.F. for the system has 
been obtained in case failures follow exponential time distribution. A numerical example together with its graphical illustration has also been appended at the last to highlight important results of the study.

Fig-2 is the graph showing the reliability of the system at any time ' $t$ ', when failures follow Weibull and Exponential time distributions. A deep study of this graph yields that the reliability of the complex system decreases approximately at a uniform rate in case of exponential time distribution whereas it decreases very rapidly in case failures follow Weibull time distribution.

Again fig-3 is the graph showing the M.T.T.F. of the system for various values of failure rates. An examination of this graph reveals that M.T.T.F. decreases catastrophically in the beginning but thereafter it decreases in uniform manner.

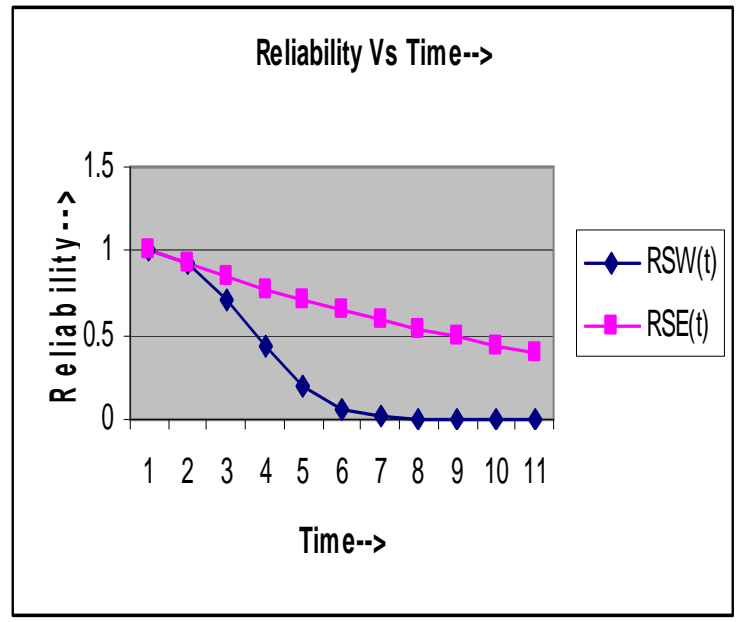

Fig-2

\section{REFERENCES}

[1] Barlow, R.E; Proschan, F. (1965): "Mathematical Theory of Reliability", New York; John Wiley.

[2] Chung, W,K (1988): “ A K-out-of-n:G redundant system with dependant failure rates and common cause failures", Microelectron. Reliability U.K., vol 28,PP 201-203.

[3] Gnedenko, B.V; Belayer, Y.K; Soloyar(1969) : "Mathematical Methods of Reliability Theory", Academic press, New York.

[4] Gupta, P.P.; Gupta, R.K.(1986): “Cost analysis of an electronic repairable redundant system with critical human errors", Microelectron . Reliab. , U.K, vol 26, PP 417-421.

[5] Nagraja, H.N.; Kannan, N.; Krishnan, N.B.(2004): "Reliability", Springer Publication.

[6] Pandey,D; Jacob , Mendus (1995) : “ cost analysis ,availability and MTTF of a three state standby complex system under common-cause and human failures", Microelectronic . Reliab., U.K., vol. 35, PP 91-95.

[7] Sharma, S.K. ;Sharma, Deepankar ; Masood, Monis: "Availability estimation of urea manufacturing fertilizer plant with imperfect switching and environmental failure", Journal of combinatorics,

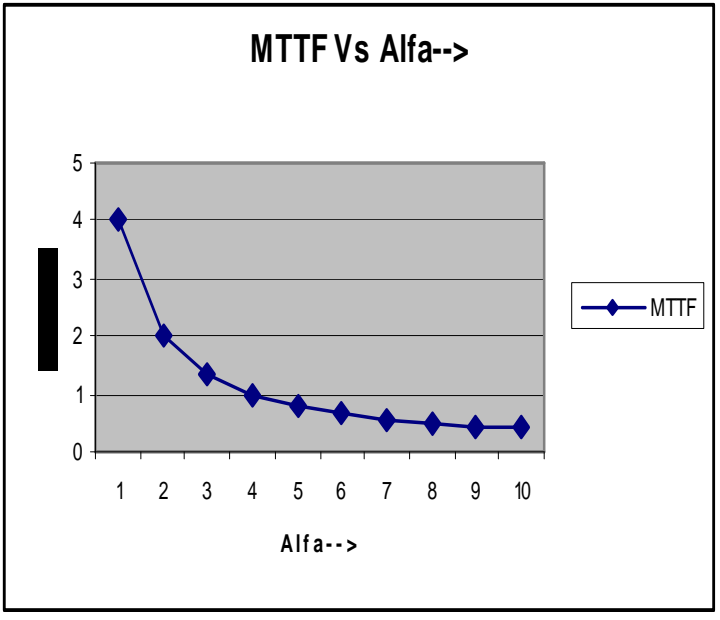

Fig-3 information \& system sciences, Vol.29, Nos. 1-4, pp135-141(2005).

[8] Sharma, Deepankar, Sharma, Jyoti ; "Estimation of reliability parameters for telecommunication system", Journal of combinatorics, information \& system sciences, Vol.29, Nos. 1-4, pp151-160(2005).

[9] Sharma, Deepankar; Goel, C.K. ; Sharma, Vinit : "Reliability and MTTF evaluation of telecommunication system", Bulletin of pure and applied Sciences, Vol.24 (E), No.2, pp349-354 (2005). 